\title{
Case 65 Hypothalamic Hamartoma
}

Abdulrahman J. Sabbagh, Sandeep Mittal, Fahad Eid Alotaibi, and José Luis Montes

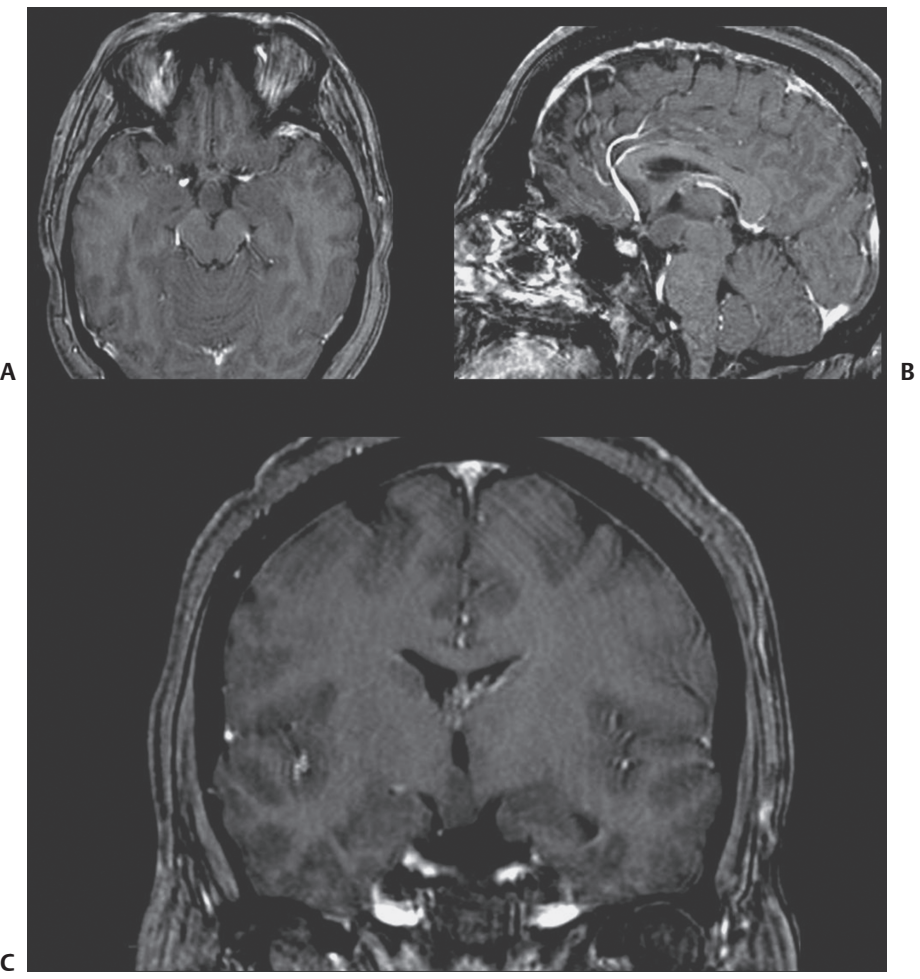

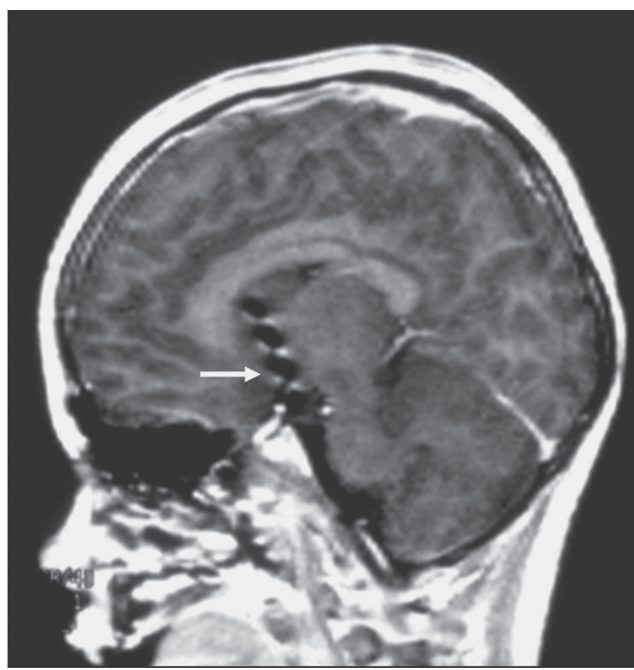

Fig. 65.2 T1-weighted sagittal magnetic resonance images of the brain showing depth electrode within the hypothalamic hamartoma.

Fig. 65.1 T1-weighted magnetic resonance images of the brain with contrast, relevant (A) axial, (B) sagittal, and (C) coronal slices are shown.

\section{Clinical Presentation}

- A 16-year-old boy is referred to you by an epileptologist. He presents with a history of progressive epilepsy that is refractory to medication.

- The seizures are described as episodes of short bouts of emotionless laughter with loss of awareness that last only a few seconds at a time and occur several times during the day now.
- He also suffers from generalized tonic-clonic seizures several times a month.

- Other pertinent findings include cognitive delay. He is able to speak. The remainder of his neurologic exam is normal (including motor, sensory, cerebellar, and gait examination).

- A magnetic resonance imaging (MRI) scan is obtained and shown in Fig. 65.1.

\section{Questions}

1. Describe the MRI (Fig. 65.1).

2. What is the term used for type of seizures this patient is experiencing?

3. How will you work up this patient?

4. Briefly describe the anatomy of the hypothalamus; enumerate its nuclei and their functions.

5. What seizure types are associated with hypothalamic hamartomas $(\mathrm{HH})$ ?
6. What symptoms other than epilepsy are associated with $\mathrm{HH}$ ?

7. If this patient has polydactyly and hypopituitarism, what syndrome would you want to confirm?

8. How do you classify HH? What class does this patient's hamartoma belong to?

9. What treatment options can you offer to a patient with $\mathrm{HH}$ and intractable seizures? What are their limitations and outcomes? 


\section{Answers}

1. Describe the MRI (Fig. 65.1).

- MRI shows a small nonenhancing, isointense lesion in the hypothalamic area.

- The mass occupies the 3rd ventricle and does not cause hydrocephalus.

- This is most consistent with a HH.

2. What is the term used for type of seizures this patient is experiencing?

- Gelastic seizures

3. How will you work up this patient?

- Imaging and electrophysiologic investigations include

- Electroencephalogram (EEG): can show slow spike and wave EEG patterns with or without multifocal epileptiform abnormalities (typically frontal or temporal $)^{1}$

- Computed tomography (CT) scan: may show an isodense nonenhancing lesion

- MRI: shows an isotense to slightly hypointense lesion compared with gray matter ${ }^{2}$

- Depth electrode recording: When the diagnosis is equivocal and EEG is nonspecific, specialized centers may consider this modality for diagnosis (Fig. 65.2). ${ }^{3,4}$

- Positron emission tomography scan: reveals ictal hypermetabolism at the hamartoma site $^{5}$

- Single photon emission computerized tomography (SPECT) imaging: measures regional cerebral blood flow during seizures. Ictal SPECT scans can be done after injection of the tracer technetium-99m hexamethylpropyleneamine oxime (Tc-99m-HMPAO).

- Magnetoencephalography (MEG): MEG maps interictal magnetic dipole sources onto MRI to produce a magnetic source image ${ }^{6}$

- Magnetic resonance spectroscopy: decrease in $\mathrm{N}$-acetyl aspartate/creatine and an increase in myoinositol/creatine $(\mathrm{mI} / \mathrm{Cr}$ ) ratios in tumor tissue when compared with values in normal gray matter of the amygdala. Choline/creatine ratios were also increased when compared with those in normal gray matter controls ${ }^{7}$

- Endocrinologic workup

- See Case 12, Pituitary Adenoma, for details

4. Briefly describe the anatomy of the hypothalamus; епumerate its nuclei and their functions.
- The hypothalamus is commonly subdivided into regions along its anteroposterior axis (Fig. 65.3). ${ }^{8}$

- The preoptic region extends rostrally to the optic chiasm and dorsally to the anterior commissure.

- The supraoptic region resides above the optic chiasm.

- The tuberal region lies above and includes the tuber cinereum.

- The mammillary region includes the mammillary bodies and the posterior hypothalamic nuclei.

5. What seizure types are associated with $\mathrm{HH}$ ?

- Seizure types include

- Gelastic seizures (forced bouts of emotionless laughter) are considered by most authors to be characteristic of $\mathrm{HH}$.

- Multiple other seizure types exist including

- Generalized tonic-clonic seizures

- Partial complex seizures

- Drop attacks

- Atypical absences

6. What symptoms other than epilepsy are associated with $\mathrm{HH}$ ?

- Other associated symptoms can include

- Precocious puberty

- Psychiatric manifestations $s^{9,10}$

- Oppositional defiant disorder (83.3\%)

- Attention deficit-hyperactivity disorder (75\%)

- Conduct disorder (33.3\%)

- Affective disorders (16.7\%)

- Progressive cognitive decline

7. If this patient has polydactyly and hypopituitarism, what syndrome would you want to confirm?

- Pallister-Hall syndrome (PHS) ${ }^{11}$

- The syndrome is typically characterized by the presence of a $\mathrm{HH}$ in association with multisystem malformations.

- The spectrum of features also includes pituitary hypoplasia or dysfunction, central postaxial polydactyly, dysplastic nails, bifid epiglottis, and imperforate anus.

- Additionally, cardiac anomalies, renal defects, and mild mental retardation are seen.

- PHS is often diagnosed at birth. In familial cases it is inherited in an autosomal dominant pattern with variable expressivity. 


\section{Answers (continued)}

\section{Hypothalamic Nuclei - Anatomy and Function}

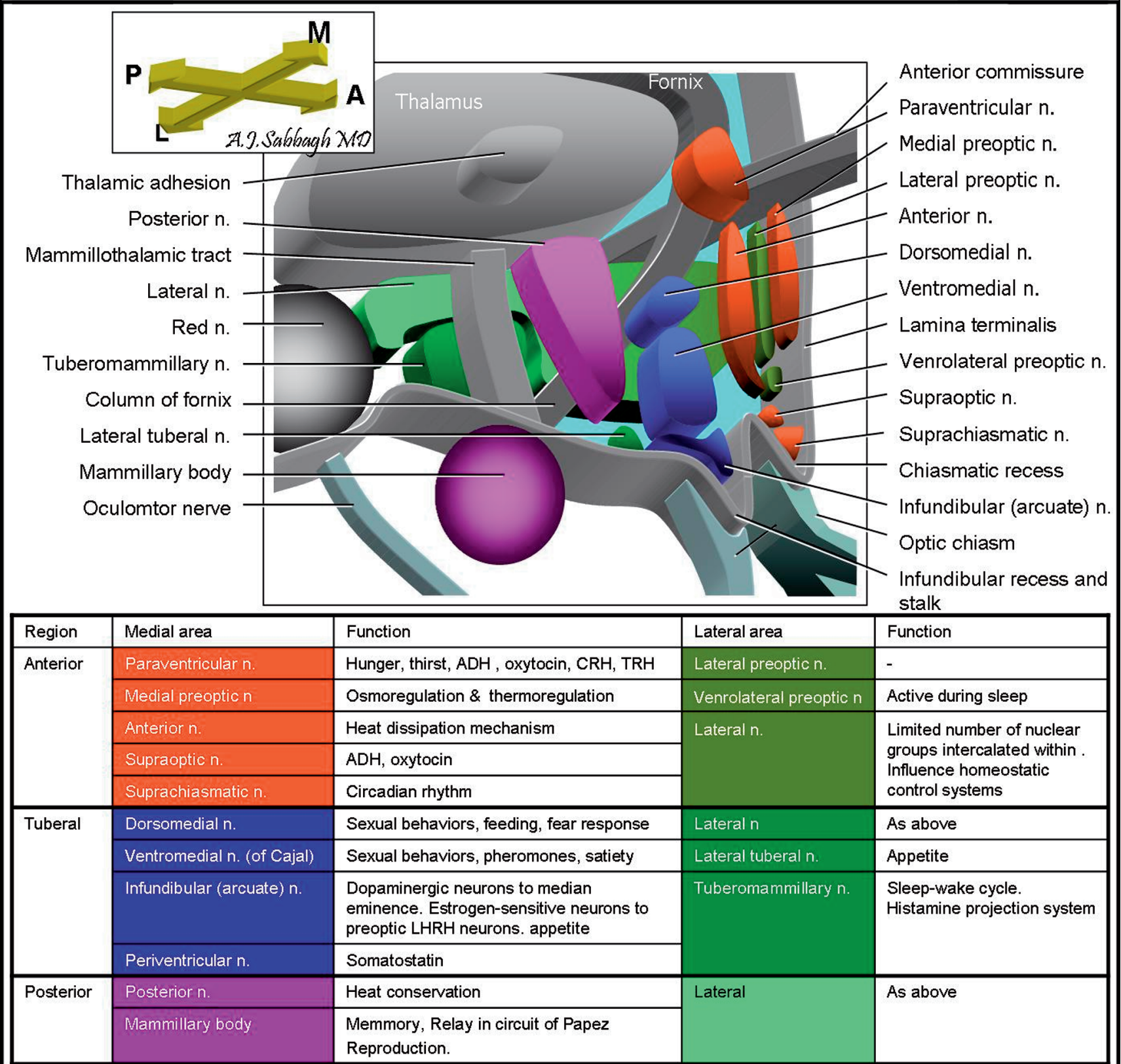

\section{A.J.Sabbagh MD}

Fig. 65.3 Hypothalamic nuclei and regions. Medial and lateral areas are illustrated in shades of red/purple or green, respectively. n, nucleus; $A D H$, antidiuretic hormone; $\mathrm{CRH}$, corticotrophin-releasing hormone; TRH, thy- rotropin-releasing hormone; LHRH, luteinizing hormone release hormone; A, anterior; P, posterior; M, medial; L, lateral. (Courtesy of the Pan Arab Journal of Neurosurgery.) 


\section{Answers (continued)}
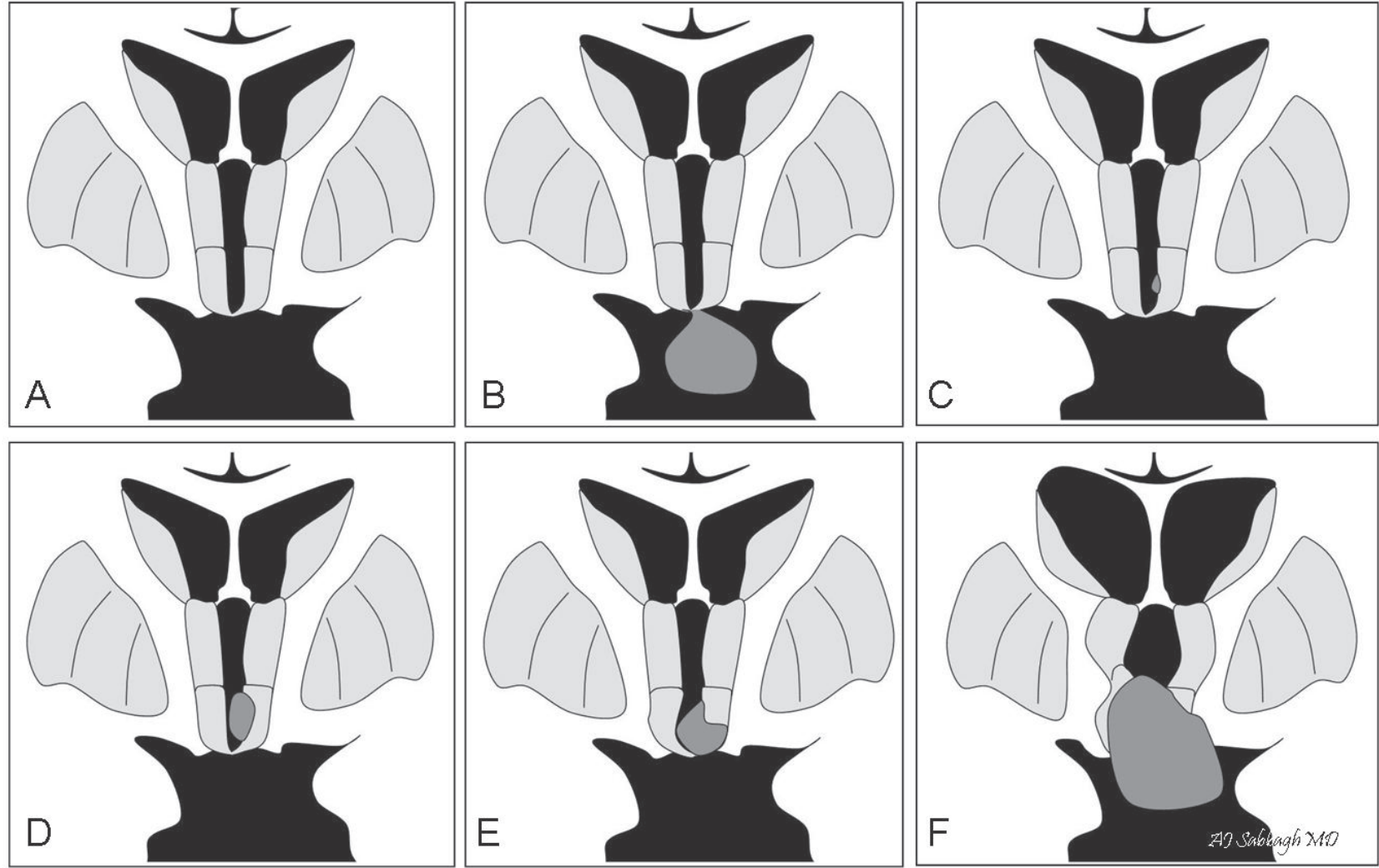

Fig. 65.4 Classification of hypothalamic hamartomas (Coronal section). (A) Normal; (B) peduncular, Delalande \& Fohlen type I (horizontal insertion); (C) parahypothalamic, sessile Delalande \& Fohlen type II (vertical insertion); (D) intrahypothalamic, sessile, Delalande \& Fohlen type III (vertical insertion); (E) intrahypothalamic, Delalande \& Fohlen type III

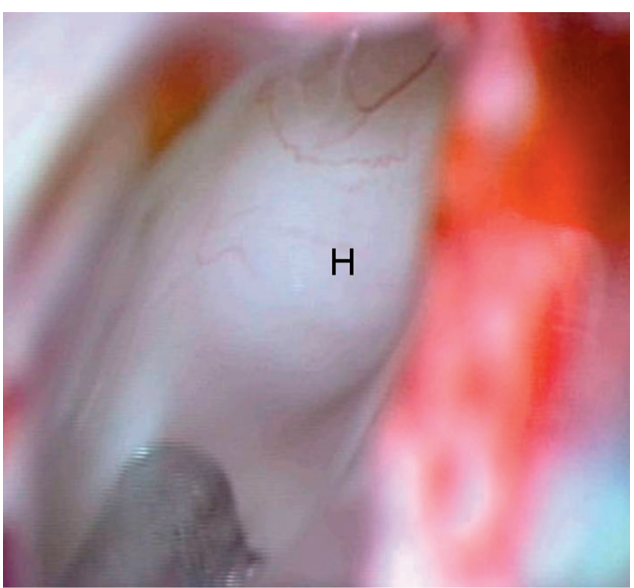

Fig. 65.5 Intraoperative endoscopic view of a hypothalamic hamartoma $(\mathrm{H})$. (horizontal and vertical insertion); (F) intrahypothalamic, Delalande \& Fohlen type IV (giant). (Adapted from Delalande O, Fohlen M. Disconnecting surgical treatment of hypothalamic hamartoma in children and adults with refractory epilepsy and proposal of a new classification. Neurol Med Chir (Tokyo) 2003;43(2):61-68.) 


\section{Answers (continued)}

8. How do you classify HH? What class does this patient's hamartoma belong to?

- Several classification schemes have been described.

- The most recent and most widely used is the classification of Delalande and Fohlen (Fig. 65.4). ${ }^{12}$

- The present patient's lesion is classified as intrahypothalamic (3E) according to Delalande and Fohlen's classification.

9. What treatment options can you offer to a patient with $\mathrm{HH}$ and intractable seizures? What are their limitations and outcomes?

- Microsurgical resection: Seizure outcome is related to completeness of resection. ${ }^{13,14}$

- Pterional and frontotemporal approach ${ }^{14}$

- Advantage: shortest, most direct route to the suprasellar cistern and hamartoma

- Disadvantage: Surgical corridor may be narrowed by the internal carotid artery, optic nerve and tracts, oculomotor nerve, and pituitary stalk.

- Outcome: $23 \%$ of patients are seizure free, $87 \%$ have significant seizure reduction (in a study of 13 patients).

- Transcallosal interforniceal approach ${ }^{15}$

- Advantage: provides a wide exposure to the 3rd ventricle and an excellent view of the hamartoma from above; avoidance of cranial nerves and blood vessels in the suprasellar cistern and interpeduncular fossa may further reduce the risk of stroke and oculomotor nerve injury

- Disadvantage: risk of short-term memory deficits because of potential septal, forniceal, or mammillary body injury

- Outcome: $52 \%$ are seizure free, $24 \%$ have significant improvement (in a series of 29 patients) ${ }^{15}$

- Complications: thalamic infarct in $7 \%$, increased appetite in $33 \%$ (this is permanent in 16\%), and short-term memory deficits in $50 \%$

- Transcallosal, subchoroidal approach: alternative to the transcallosal interforniceal route; lower risk of short-term memory deficits

- Endoscopic transventricular approach (Fig. 65.5 for an endoscopic view)

- Outcome: $31 \%$ were able to sustain complete resection (14 of 44 patients), of whom $90 \%$ were seizure free. ${ }^{16}$

- Complications: short-term memory difficulties (three patients) and hemiparesis (one patient). ${ }^{16}$
- Disconnection procedure (open or endoscopic) ${ }^{17}$

- Outcome: $58 \%$ seizure free

- Complications (in a series of 18 patients) ${ }^{17}$ : stroke ( 2 patients), diabetes insipidus ( 2 patients), meningitis ( 1 patient)

- Stereotactic radiosurgery ${ }^{18}$

- Gamma knife radiosurgery: good treatment for small- and medium-size hamartomas. The median dose recommended at the marginal isodose is 17 Gy (range is $13-26 \mathrm{~Gy}$ ).

- Outcome: $37 \%$ seizure free. Most had cognitive and behavioral improvement.

- Complications: $15 \%$ transient worsening of seizures; no permanent complications mentioned.

- Linear acceleration-based radiosurgery

- Stereotactic brachytherapy

- Stereotactic radiofrequency ablation

- Outcome: $25 \%$ seizure free, $25 \%$ significant improvement (12 patients) $)^{19}$

- Complications: $8 \%$ mortality (1 patient), 16\% memory deficits

- Vagal nerve stimulation: limited role; palliative and seizure freedom is not expected. ${ }^{20}$

- Corpus callosotomy: limited role 


\section{References}

1. Berkovic SF, Andermann F, Melanson D, Ethier RE, Feindel W, Gloor P. Hypothalamic hamartomas and ictal laughter: evolution of a characteristic epileptic syndrome and diagnostic value of magnetic resonance imaging. Ann Neurol 1988;23(5):429-439

2. Hahn FJ, Leibrock LG, Huseman CA, Makos MM. The MR appearance of hypothalamic hamartoma. Neuroradiology 1988;30(1):65-68

3. MunariC, KahaneP, Franciones, . Role of the hypothalamic hamartoma in the genesis of gelastic fits (a video-stereo-EEG study). Electroencephalogr Clin Neurophysiol 1995;95(3):154-160

4. Palmini A, Chandler C, Andermann F, et al. Resection of the lesion in patients with hypothalamic hamartomas and catastrophic epilepsy. Neurology 2002;58(9):1338-1347

5. Palmini A, Van Paesschen W, Dupont P, Van Laere K, Van Driel G. Status gelasticus after temporal lobectomy: ictal FDG-PET findings and the question of dual pathology involving hypothalamic hamartomas. Epilepsia 2005;46(8):1313-1316

6. Tovar-Spinoza ZS, Ochi A, Rutka JT, Go C, Otsubo H. The role of magnetoencephalography in epilepsy surgery. Neurosurg Focus 2008;25(3):E16

7. Amstutz DR, Coons SW, Kerrigan JF, Rekate HL, Heiserman JE. Hypothalamic hamartomas: correlation of MR imaging and spectroscopic findings with tumor glial content. AJNR Am J Neuroradiol 2006;27(4):794-798

8. Parent A. Carpenter's Human Neuroanatomy. Baltimore: Williams \& Wilkins; 1996

9. Savard G, Bhanji NH, Dubeau F, Andermann F, Sadikot A. Psychiatric aspects of patients with hypothalamic hamartoma and epilepsy. Epileptic Disord 2003;5(4):229-234

10. Weissenberger AA, Dell ML, Liow K, et al. Aggression and psychiatric comorbidity in children with hypothalamic hamartomas and their unaffected siblings. J Am Acad Child Adolesc Psychiatry 2001;40(6):696-703

11. Hall JG, Pallister PD, Clarren SK, et al. Congenital hypothalamic hamartoblastoma, hypopituitarism, imperforate anus and post- axial polydactyly-a new syndrome? Part I: clinical, causal, and pathogenetic considerations. Am J Med Genet 1980;7(1):47-74

12. Delalande O, Fohlen M. Disconnecting surgical treatment of hypothalamic hamartoma in children and adults with refractory epilepsy and proposal of a new classification. Neurol Med Chir (Tokyo) 2003;43(2):61-68

13. Mittal S, Montes JL, Farmer JP, Sabbagh AJ. Surgical management of epilepsy related to hypothalamic hamartomas. In: Villemure JG, Baltuch G, eds. Operative Techniques in Epilepsy Surgery. New York: Thieme Medical Publishers; 2009:81-98

14. Palmini A, Paglioli-Neto E, Montes J, Farmer JP. The treatment of patients with hypothalamic hamartomas, epilepsy and behavioural abnormalities: facts and hypotheses. Epileptic Disord 2003;5(4):249-255

15. Harvey AS, Freeman JL, Berkovic SF, Rosenfeld JV. Transcallosal resection of hypothalamic hamartomas in patients with intractable epilepsy. Epileptic Disord 2003;5(4):257-265

16. Rekate HL, Feiz-Erfan I, Ng YT, Gonzalez LF, Kerrigan JF. Endoscopic surgery for hypothalamic hamartomas causing medically refractory gelastic epilepsy. Childs Nerv Syst 2006;22(8):874-880

17. Fohlen M, Lellouch A, Delalande O. Hypothalamic hamartoma with refractory epilepsy: surgical procedures and results in 18 patients. Epileptic Disord 2003;5(4):267-273

18. Régis J, Scavarda D, Tamura M, et al. Epilepsy related to hypothalamic hamartomas: surgical management with special reference to gamma knife surgery. Childs Nerv Syst 2006;22(8):881-895

19. Kuzniecky RI, Guthrie BL. Stereotactic surgical approach to hypothalamic hamartomas. Epileptic Disord 203;5(4):275-280

20. Feiz-Erfan I, Horn EM, Rekate HL, et al. Surgical strategies for approaching hypothalamic hamartomas causing gelastic seizures in the pediatric population: transventricular compared with skull base approaches. J Neurosurg 2005; 103(4, Suppl):325-332 